\title{
CUE: The continuous unified electronic diary method
}

\author{
Kate Ellis-Davies • Elena Sakkalou • Nia C. Fowler • \\ Elma E. Hilbrink $\cdot$ Merideth Gattis
}

Published online: 31 May 2012

(C) Psychonomic Society, Inc. 2012

\begin{abstract}
In the present article, we introduce the continuous unified electronic (CUE) diary method, a longitudinal, event-based, electronic parent report method that allows real-time recording of infant and child behavior in natural contexts. Thirty-nine expectant mothers were trained to identify and record target behaviors into programmed handheld computers. From birth to 18 months, maternal reporters recorded the initial, second, and third occurrences of seven target motor behaviors: palmar grasp, rolls from side to back, reaching when sitting, pincer grip, crawling, walking, and climbing stairs. Compliance was assessed as two valid entries per behavior: $97 \%$ of maternal reporters met compliance criteria. Reliability was assessed by comparing diary entries with researcher assessments for three of the motor behaviors: palmar grasp, pincer grip and walking. A total of $81 \%$ of maternal reporters met reliability criteria. For those three target behaviors, age of emergence was compared across data from the CUE diary method and researcher assessments. The CUE diary method was found to detect behaviors earlier and with greater sensitivity to individual differences. The CUE diary method is shown to be a reliable methodological tool for studying processes of change in human development.
\end{abstract}

Keywords Electronic diary · Infancy · Child ·

Motor development · Ecological momentary assessment . Ambulatory assessment $\cdot$ Parent report

A unifying aim for developmental psychology is to understand the processes of change in human behavior (Adolph \&

K. Ellis-Davies $\cdot$ E. Sakkalou $\cdot$ N. C. Fowler $\cdot$ E. E. Hilbrink

M. Gattis $(\bowtie)$

Cardiff University,

Cardiff, UK

e-mail: gattism@cardiff.ac.uk
Robinson, 2008; Kagan, 2008; Muller \& Giesbrecht, 2008). Theories of development are often contrasted in terms of their accounts of change, in particular whether processes of change are qualitative or quantitative, and continuous or discontinuous. Importantly, methodological tools often influence theoretical accounts of change by yielding data that favors one account of change over another (McCall, 1981). In the present article, we will briefly review the major methodological tools in developmental psychology and then introduce a new method-a continuous unified electronic diary method for gathering developmental data.

Experiments are systematic and rigorous methods for studying elicited behavior, typically in a laboratory setting. Because they involve the manipulation of an independent variable, experimental methods allow clear analysis of cause-effect relations and are useful for addressing questions about the relation between a specific behavioral phenomenon and a specific eliciting context (Cronbach, 1957; Danziger, 2000; Holmes \& Teti, 2008). Experimental methods are not suitable, however, for addressing questions about the spontaneous emergence of behavioral phenomena, and are of limited usefulness for addressing questions about behavior across time and context, or, in other words, behavioral change (Stone, Broderick, Kaell, DelesPaul, \& Porter, 2000). In addition, experiments are susceptible to data distortion caused by low sampling frequency (Adolph, Robinson, Young, \& Gill-Alvarez, 2008; Kuhn, 1995). As a result, experiments may miss periods of instability and change, leading to inaccurate estimates of developmental trajectories. These problems are especially critical for low-frequency and/or newly emerging behaviors. For these reasons, experimental methods have limited utility for addressing questions about processes of change.

Observations are a practical method for studying naturally occurring behavior, whether spontaneous or elicited, and whether in the laboratory, home, or daycare environment. 
Observational methods allow analysis of relations between children and the contexts in which they are developing and are useful for addressing questions about the bidirectional influences that parents and children have on one another (Bakeman, 1983; Bornstein, 1985; Danis, Bourdais, \& Ruel, 2000; Mitchell, 1979). Observational methods deliver greater contextual information and, as a result, can have greater generalizability than experimental methods. Observational methods are labor intensive, however, and are prone to the same problems of sampling frequency as experimental methods, with the result that they too have limited utility for addressing questions about processes of change.

Parent reports are a form of observation that rely on parents as observers and reporters of behavior, according to their day-to-day experience with a child. The most common forms of parent report are checklists and questionnaires (Dekker, Nunn, Einfeld, Tonge, \& Koot, 2002; Dunn, 1990; Fenson et al., 2000; Gartstein \& Rothbart, 2003; Reznick \& Goldfield, 1992). Because they draw on parents' regular experience with their children, checklists and questionnaires are less susceptible to some of the problems of sampling frequency associated with experimental and observational methods. Because parent-report methods are usually retrospective, however, they are susceptible to other sources of data distortion (Seifer, 2005), in particular parents' abilities to recall behaviors that have occurred in the past (Green, Rafaeli, Bolger, Shrout, \& Reis, 2006; Reznick \& Goldfield, 1994). Importantly, most studies using checklists and questionnaires have limited options for assessing parents' compliance or reliability as reporters. In addition, because checklists and questionnaires are administered at a specific moment in time, they are of limited utility for identifying emergence or change in behaviors. Most checklists and questionnaires also lack critical contextual information such as social and physical context, which in turn limits their utility for addressing questions about processes of change in development.

Methodologically, parent-report diaries address many of the limitations of the experimental and observational methods discussed above. Because parent-report diaries are based on continuous observation, they provide a high sampling rate over a specified period, and, in addition, they offer data about children in their natural environment (e.g., Callanan \& Oakes, 1992; Johnson \& Mervis, 1997; Naigles, Hoff, \& Vear, 2009; Nelson, 1989; Pine, 1995; Robinson \& Mervis, 1999; Tomasello, 1992; Wolfson, Lacks, \& Futterman, 1992). Even the most rigorous schedule of experimental testing can miss critical points in development because of sampling distortion (Adolph et al., 2008; Lewis, 2000). By comparison, the eventbased data from diaries reveal patterns of behavior and development with less sampling skew and distortion (Bolger, Davis, \& Rafaeli, 2003). Parent-report diaries thus have the potential to yield extremely valuable developmental data, including information on age of emergence, age of skill progression, and contextual variables such as precursor variables, eliciting factors, and rewards.

Diary studies have, however, historically lacked the rigor and systematic strengths of experimental methods. In particular, previous studies involving parent-report diaries have frequently had very small sample sizes, high attrition, and lacked sufficient assessment of compliance and reliability (Stone, Shiffman, Schwartz, Broderick, \& Hufford 2002; Miller, 1981; Naigles et al., 2009). Until recently, the only method for collecting parent-report diaries was paper and pencil, with the result that reporting was an onerous task, and it was not possible to monitor when entries were made or to assess compliance with reporting instructions, such as entering reports at requested times. Reliability of reporters has also been a concern with diary methods. Low reliability may result from insufficient training of reporters to observe and record behaviors; from the onerous nature of paper-andpencil diaries, which may lead to fewer and/or more retrospective reports, or simply from parental biases in reporting.

Over the past decade, psychologists and social scientists have increasingly turned to electronic diaries to address these problems while at the same time exploiting the many advantages of diary data (Green et al., 2006). Electronic diaries allow for easier and more consistent reports across participants (Hufford \& Shields, 2002). For example, in one study comparing paper and electronic diary reports of infant fussiness and caregiver holding, maternal reporters described paper diaries as more onerous and made more frequent entries with electronic diaries (Lam et al., 2010). In addition, because electronic diaries contain automatic timestamp information for entries, they allow researchers to monitor and assess participant entries for compliance and reliability (Ebner-Priemer \& Trull, 2009). Electronic diaries, sometimes referred to as ecological momentary assessment or ambulatory assessment, are now increasingly used by researchers investigating psychological phenomena such as emotional functioning, mood disorders, stress, organizational behavior, and health treatments (Alpers, 2009; Gunthert, Conner, Armeli, Tennen, Covault, \& Kranzler, 2007; EbnerPriemer et al., 2007; Ebner-Priemer, \& Sawitzki, 2007; Fahrenberg, Myrtek, Pawlik, \& Perrez, 2007; Myrtek, Aschenbrenner, \& Brugner 2005; Hoppmann \& Riediger, 2009; Jamison et al., 2001; King, Lluch, Stubbs, \& Blundell, 1997; Klumb, Elfering, \& Herre, 2009).

In the present article, we introduce a new event-based adaptation of electronic diaries intended for use by developmental psychologists. Electronic diaries are a potentially valuable methodological tool for developmental psychologists because they draw on continuous observation and allow the study of behavior in natural contexts, while at the same time offering consistency and ease of use across reporters, as well as assessment of compliance and reliability. Most studies using electronic diary data collection have 
involved self-report, however, whereas collecting data on infants and children requires another person, usually a parent, to do the reporting.

The CUE diary method has two components. First, we developed a reporting method suitable for parent reporters that yields data containing sufficient contextual information to be useful to developmental psychologists interested in change processes. We used the Experience Sampling Programme (ESP.4) (Feldman Barrett \& Barrett, 2001) to develop a series of event-based questions that allowed parents to identify and record target behaviors on a continuous basis. The questions were designed to elicit accurate behavioral observations with appropriate contextual information, and at the same time to ensure ease of use and consistency of reporting across parents. To do so, we used a combination of open-ended questions with free-text response fields and closed-class questions with categorical answers available from a drop-down menu. The complete CUE diary method addresses the development of communicative, imitative, and motor behaviors, but in the present report, we focus on motor behaviors only, since they were critical to our assessment of the method (see Fig. 1).

Second, we developed a training and support system to ensure that parent reporters knew how to use the electronic diary, had sufficient understanding of the target behaviors to be able to identify them accurately, and were able to remember what was requested of them. This was an important step in adapting electronic diary methods to the study of human development, since electronic diaries have previously been used primarily for self-report rather than the reporting of parent-observed infant and child behaviors. To provide training and support for parent-observed recording of developmental phenomena, we recruited mothers into the study during pregnancy, trained them to identify and record infant behaviors, and gave them supporting materials to take home. Mothers were given clear definitions of target behaviors and were instructed to record the first three occurrences of each behavior as soon as possible after it was observed. Mothers were asked to identify and record target developmental phenomena from birth to 18 months. To help maintain accuracy and motivation throughout that period, maternal reporters also attended monthly assessment meetings and were given monthly feedback on a separate occasion.

To test the utility of the CUE diary method, we evaluated compliance, reliability, and validity of maternal reporters. To evaluate compliance, we examined reporting of three target motor behaviors: palmar grasp, pincer grip, and walking. We chose these three behaviors because they span the complexity of motor development over infancy, where the palmar grasp is a reflex, pincer grip is a fine motor behavior, and walking is a gross motor behavior. Compliance evaluation focused on whether behaviors were accurately identified (or, in other words, were consistent with the provided definition), had been recorded at least twice, and had been recorded as soon as possible after observation.

To evaluate the reliability of the CUE diary method as well as the reliability of individual reporters, we compared CUE diary data to researcher assessments of the three target motor behaviors (palmar grasp, pincer grip, and walking). Reliability evaluation focused on whether maternal reporters identified a behavior at least as soon as a researcher identified it, and not more than two months before.

Having developed an event-based electronic diary method for the study of infant and child development, we then wanted to demonstrate the value of the data yielded by this method. To do so, we first analyzed data about onset for the seven motor behaviors of palmar grasp, rolls from side to back, reaches when sitting, pincer grip, crawling, walking, and climbing stairs. These behaviors span the first 18 months of life, encompassing reflex, fine, and gross motor development. Secondly, we compared age-of-emergence for our three target motor behaviors of palmar grasp, pincer grip, and walking, from CUE diary data and researcher-assessed data, to examine whether electronic diary data would be less prone to data distortion. Because reflex and fine motor behaviors involve fewer components, we expected it would be easier for maternal reporters to identify such behaviors accurately. By comparison, gross motor behaviors involve multiple components, and we therefore expected that it would be more difficult for maternal reporters to identify them accurately. We also expected that the continuous, event-based sampling of the CUE diary method would yield estimations of developmental trajectories without the sampling distortion of researcher-assessed data.

The present study is the first step of a larger study using the CUE diary method to investigate interactions among cognitive, communicative, and motor development. Evaluating compliance and reliability was critical to our study because doing so not only allows us to establish the validity of the method, but also to identify and exclude unreliable reporters at subsequent steps in the larger study.

\section{Method}

\section{Participants}

Forty expectant mothers were recruited during their last trimester of pregnancy to participate in First Steps, a longitudinal study of infant development from birth until 18 months. Expecting parents were recruited from community organizations within Cardiff. The First Steps design involved continuous electronic diary reporting from birth to 18 months, and monthly testing from 2 until 18 months. At each monthly testing session, families were given $£ 25$ in shopping vouchers and a baby gift, such as a toy, t-shirt, or book, in return for their participation. As an incentive to 


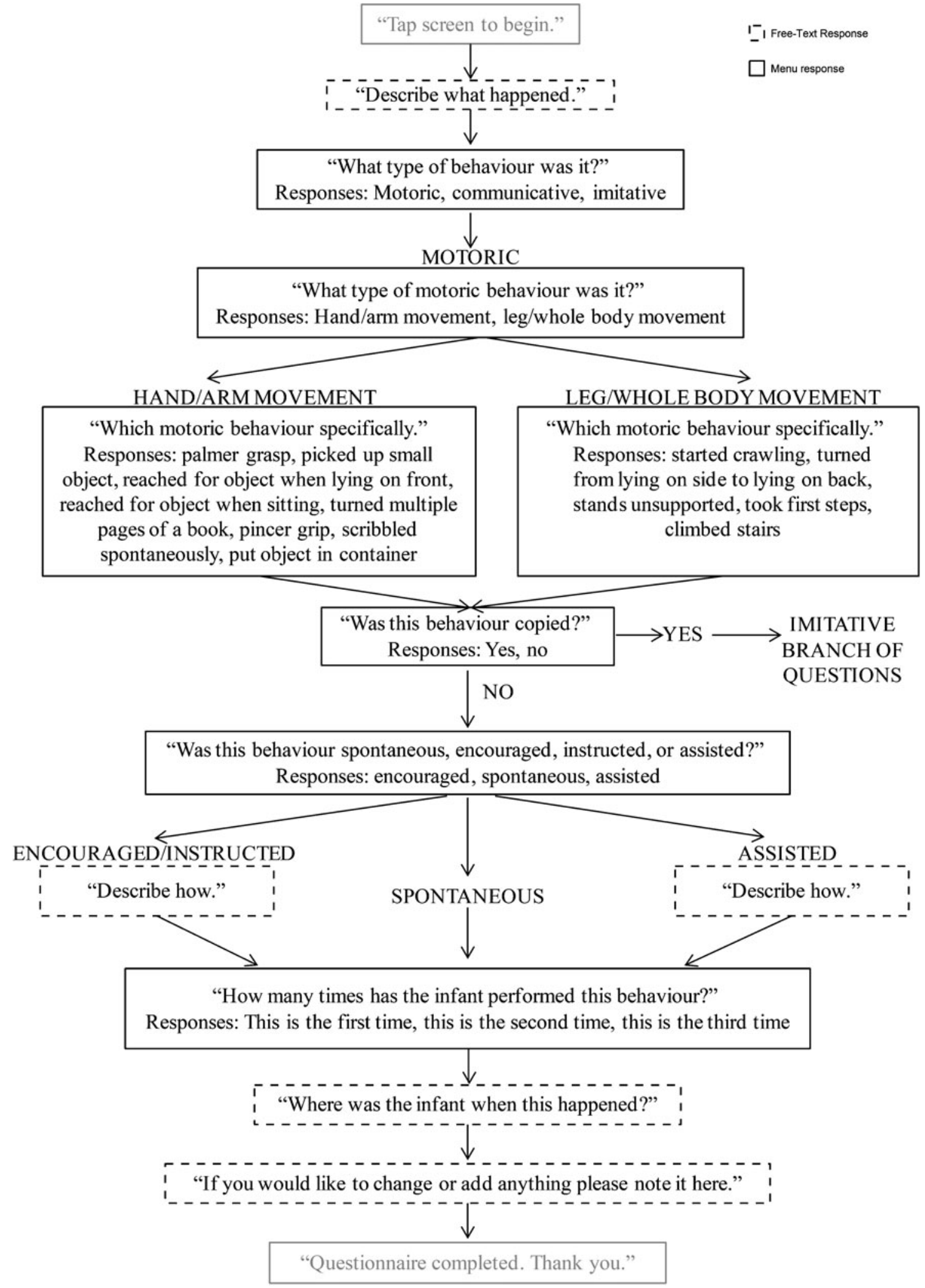

Fig. 1 Questions on motor development used in continuous unified electronic (CUE) diary method. Greeting and exit messages are shown in grey. Questions had either free-text responses (dashed line) or menu responses (solid line) 
complete the study, families were given an additional 2250 in shopping vouchers at the end of the study. The sample of reporters ranged in age, nationality, ethnicity, education level, socioeconomic status, marital status, and the number of previous children, as shown in Table 1. One mother did not begin the study. Of the remaining 39 mother-infant dyads, all infants were born to term with 18 females and 21 males.

The Cardiff University School of Psychology Research Ethics Committee and the South East Wales Local Research Ethics Committee of the Cardiff and Vale National Health Service Trust both reviewed the study protocol and granted ethical approval. Each maternal reporter gave informed consent prior to participation in the study.

\section{Electronic diary}

Palm Pilot Z20s were installed with Version 4 of the Experience Sampling Programme (ESP.4) (Feldman Barrett \& Barrett, 2001). ESP.4 is free, open-source software for collecting questionnaire data electronically. The software presents questions and records answers and response times (RTs) and creates an automatic time stamp for each entry. Once a question is completed, the software proceeds to the next question automatically. To prevent participants from changing entries post hoc, the software does not allow reviewing or changing of entries. For analysis, data from individual handheld devices can be uploaded onto a central computer.

A series of event-based questions were created in ESP that allowed parents to identify and record communicative, imitative, and motor behaviors on a continuous basis (see Fig. 1). Because our assessment of the reliability and validity of the method focused on motor behaviors, only motor behaviors are reported here. Some questions were open-ended and had freetext response fields, whereas other questions were closed class and had categorical answers available from a drop-down menu. Each entry thus identified a particular infant behavior and various contextual variables, including the social setting, the physical setting, whether the behavior was elicited or spontaneous, and whether this was the first, second, or third occasion on which the behavior occurred. Sampling was event based and therefore determined by the frequency of infant behavior, rather than in response to a time-based prompt from the handheld device.

\section{Procedure}

Initial training and support material During the last trimester of pregnancy, maternal reporters participated in an introductory training session. During the session, a researcher demonstrated the CUE diary method, asked the mother to create practice entries, and gave the mother a study notebook with supporting materials (see Appendices A and B for examples). An important component of the study notebook was the first What to Expect (WTE) sheet (see Appendix C for an abbreviated example). Each WTE sheet provided a brief summary of infant development during the specified period and identified target behaviors for maternal reporters to observe and record. WTE sheets with new target behaviors were provided in 3-month intervals so that mothers did not need to hold a large number of target behaviors in mind at one time. Further training was provided with each WTE sheet. At the end of the training session, maternal reporters were instructed to create further practice entries at home.

Maternal reporters were instructed to begin reporting from the birth of their infant, and to enter each target behavior as soon as possible after it occurred. ESP creates an automatic time-stamp for each entry, including both date and time. If mothers reported an event that had occurred on a previous day, they had to enter the date it had occurred. This allowed us to evaluate entries on the basis of

Table 1 Demographic characteristics of maternal reporters, presented as a percent of each group

\begin{tabular}{llll}
\hline Maternal Reporter Characteristics & Total Sample $(\%)(N=36)$ & Excluded Sample $(\%)(N=7)$ & Final Sample $(\%)(N=29)$ \\
\hline Age in years at recruitment into study & & & 14.29 \\
$16-20$ & 2.78 & 0 & 0 \\
$21-25$ & 13.89 & 14.29 & 17.24 \\
$26-30$ & 11.11 & 28.57 & 10.34 \\
$31-35$ & 36.11 & 42.86 & 37.93 \\
$36-40$ & 36.11 & & 34.48 \\
Highest education level attained & & 28.57 & 24.14 \\
High school & 25 & 42.86 & 62.07 \\
Undergraduate degree & 58.33 & 28.57 & 13.79 \\
Postgraduate degree & 16.67 & & 58.62 \\
Birth order & & 57.14 & 41.38 \\
Primiparous & 58.33 & 42.86 & \\
Multiparous & 41.67 & & \\
\hline
\end{tabular}


compliance with this reporting requirement. In some instances, mothers kept notes in their personal diaries and created ESP entries on the basis of these notes. To ensure a clear threshold, mothers were told that recording any event more than 2 weeks after it occurred would invalidate the entry.

Monthly assessments All maternal reporters and their infants attended monthly assessments. These assessments were organized into groups, with infants allocated into a group according to the week of the month that he or she was born. This ensured that monthly visits occurred close to infants' monthly birthdays.

At each monthly assessment, diary entries were uploaded onto a researcher computer, and after the visit, entries were transferred to an online database. At this time, mothers were asked whether they had any problems with identifying or recording behaviors, or needed any clarification. At the 4-, 6-, 9-, 12-, and 15-month visits, a new WTE sheet was given to the mother, together with further training on identifying target behaviors.

At each monthly assessment, infants were also tested by a trained researcher. This testing included researcher-assessed motor milestones that formed the basis of our reliability evaluation, and other experimental measures (not reported here). As in previous parent-report diary studies, such as Bodnarchuk and Boudreau (2004), we retrospectively validated the CUE diary method and the reliability of maternal reporters by comparing the age-of-emergence of target behaviors as recorded by the maternal reporter with the age-of-emergence found in researcher assessments. These researcher assessments followed World Health Organization (WHO) methods for assessing motor development (Wijnhoven et al., 2004).

Further support and training Each maternal reporter was assigned a key researcher who was present at the introductory training session and maintained regular contact with the mother throughout the study. Following each monthly visit, key researchers reviewed the entries of maternal reporters and created a feedback sheet identifying the target behaviors for which the maternal reporter had created valid entries in the previous month and the target behaviors that the maternal reporter needed to watch for over the coming month. This monitoring and feedback system was designed to increase ease of observation for participants and to maximize reporting compliance.

\section{Results}

Compliance assessment

For an entry to be considered valid, it had to contain sufficient detail to match the target behavior definitions given in the WTE sheets (see Appendix C for an example). Entries were considered invalid if they were recorded before distribution of the WTE sheet that described the behavior, or if they were recorded more than 2 weeks after occurrence. This delay was evaluated by comparing the time stamp of each entry with the reported date of occurrence. Entries that failed to meet these criteria were excluded from analysis. Such entries made up less than $1 \%$ of the total number of entries analyzed.

Two maternal reporters failed to complete the study, and their data was excluded on this basis. One infant was referred for developmental delay and was excluded on this basis, since the instructions and training procedures were designed and piloted with typically developing infants. One maternal reporter failed to comply with the requirement of having two or more valid entries for palmar grasp, pincer grip, and walking and was excluded from the sample.

\section{Reliability assessment}

To assess reliability of the method as well as reliability of individual maternal reporters, maternal reports were compared with researcher assessments for three target motor behaviors (palmar grasp, pincer grip, and walking). For any data from a maternal reporter to be included in the final CUE diary data set, she was required to be reliable for all three of the target motor behaviors. A maternal reporter's reliability for each behavior was evaluated according to the following criteria: Each behavior must have been recorded by the maternal reporter at least by the date on which a researcher assessment identified the behavior, and each behavior must have been recorded by the maternal reporter not more than two months before the researcher assessment identified the behavior. Following the reasoning of the WHO study (Wijnhoven et al., 2004), we reasoned that because mothers have more opportunities to observe behavior, they would identify behaviors as soon as or before researchers could do so, but that we needed to have a reasonable threshold for the researcher to identify the behavior. We chose two consecutive months of testing as this threshold, reasoning that if an infant was capable of a behavior, he or she might not evidence the behavior during the first assessment, but should be able to demonstrate the behavior during at least one of two assessments. This somewhat conservative threshold was designed to ensure maximum reliability across the dataset, and in so doing to increase confidence in future analyses of CUE diary data. Seven maternal reporters failed the described reliability criteria and were excluded from the final sample of CUE data as a result. After the application of compliance and reliability criteria, $81 \%$ of maternal reporters remained. Table 1 presents demographic information for maternal reporters grouped according to inclusion status. Maternal reporters whose data were included or excluded in the final sample had similar ages, levels of education, and number of children. 
Means and other descriptives

The mean age and standard deviation for the CUE diary motor behaviors of palmar grasp, rolls from side to back, reaches when sitting, pincer grip, crawling, walking, and climbing stairs are shown in Table 2. The first column denotes the full sample before exclusion criteria were applied. The second column contains information from maternal reporters excluded on the basis of compliance and reliability criteria. The third column shows information from the final CUE sample, and the final column shows data from researcher-administered assessments. The range of age of emergence was quite small for the palmar grasp, a reflex behavior observed shortly after birth. By comparison, the range of age of emergence was larger for the pincer grip, crawling, and walking.

\section{Validity assessments}

Finally, we evaluated the CUE diary method by comparing age of emergence from CUE data for the final sample with age of emergence from researcher assessment (Columns 3 and 4 in Table 2). Researcher assessments consistently yielded higher age-of-emergence data and reduced within-group variation.

Figure 2 demonstrates the range of onset ages for all seven motor behaviors. Periods of overlap between certain behaviors, such as reaching when sitting and pincer grip, are evident. In one case, such overlap suggests reporting errors: The earliest report of climbing stairs preceded the earliest report of walking entry, a contradiction that suggests climbing stairs was not reported accurately.

Figure 3 shows a comparison between the distributions for age of emergence yielded by each method. The CUE diary data consistently produced earlier data on infants meeting milestones.

Finally, example CUE diary entries for each behavior are shown in Table 3. Within the example it is interesting to note

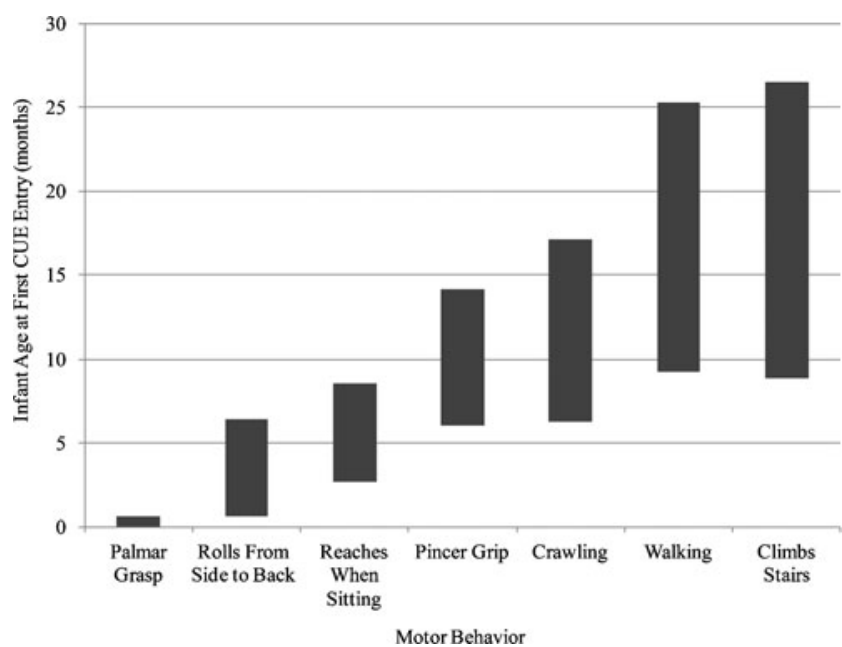

Fig. 2 Range of ages of onset for motor behaviors reported in continuous unified electronic (CUE) diary method, indicating overlap for periods of motor skill emergence. Palmar grasp, pincer grip, and walking validated via researcher-administered assessment

the contextual information provided for the fine and gross motor entries.

\section{Discussion}

We designed and evaluated an event-based, electronic maternal reporting method that allows real-time recording of infant and child behavior in natural contexts. Event-based electronic diary methods allow parents to create entries based on continuous observation, increase consistency across different entries and reporters, and allow for assessment of compliance and reliability.

Designing a continuous unified electronic diary method

Our primary aim was to design an event-based electronic diary reporting method suitable for the study of infant and

Table 2 Age of emergence for motor behaviors presented as mean $+/-S D$

\begin{tabular}{|c|c|c|c|c|c|c|c|c|}
\hline \multirow[b]{2}{*}{ Motor Behavior } & \multicolumn{2}{|c|}{ Total Sample $(N=36)$} & \multicolumn{2}{|c|}{ Excluded Sample $(N=7)$} & \multicolumn{2}{|c|}{ Final Sample $(N=29)$} & \multicolumn{2}{|c|}{ Researcher Assessment } \\
\hline & $M$ & SD & $M$ & SD & $M$ & SD & $M$ & SD \\
\hline Palmar grasp & 7.56 & 12.36 & 13.4 & 20.85 & 5.56 & 7.15 & 29 & 4 \\
\hline Rolls from side to back & 88.21 & 47.56 & 110.6 & 35.72 & 80.21 & 49.22 & $\mathrm{n} / \mathrm{a}$ & $\mathrm{n} / \mathrm{a}$ \\
\hline Reaches when sitting & 147.37 & 47.56 & 147.43 & 38.98 & 147.36 & 52.36 & $\mathrm{n} / \mathrm{a}$ & $\mathrm{n} / \mathrm{a}$ \\
\hline Pincer grip & 230.13 & 34.42 & 250.67 & 60.23 & 223.76 & 18.75 & 242 & 8 \\
\hline Crawling & 256.16 & 53.07 & 263 & 88.64 & 254.03 & 38.17 & $\mathrm{n} / \mathrm{a}$ & $\mathrm{n} / \mathrm{a}$ \\
\hline Walking & 393.49 & 59.02 & 416 & 54.04 & 387.86 & 59.78 & 411 & 57 \\
\hline Climbs stairs & 407.93 & 102.51 & 422 & 99.37 & 405.73 & 104.72 & $\mathrm{n} / \mathrm{a}$ & $\mathrm{n} / \mathrm{a}$ \\
\hline
\end{tabular}

Means are in days. The first column identifies the total sample before exclusion criteria applied. The second column denotes data for those excluded following exclusion criteria, and the third column contains the remaining final sample. The final column identifies researcher-assessed age of emergence where relevant 

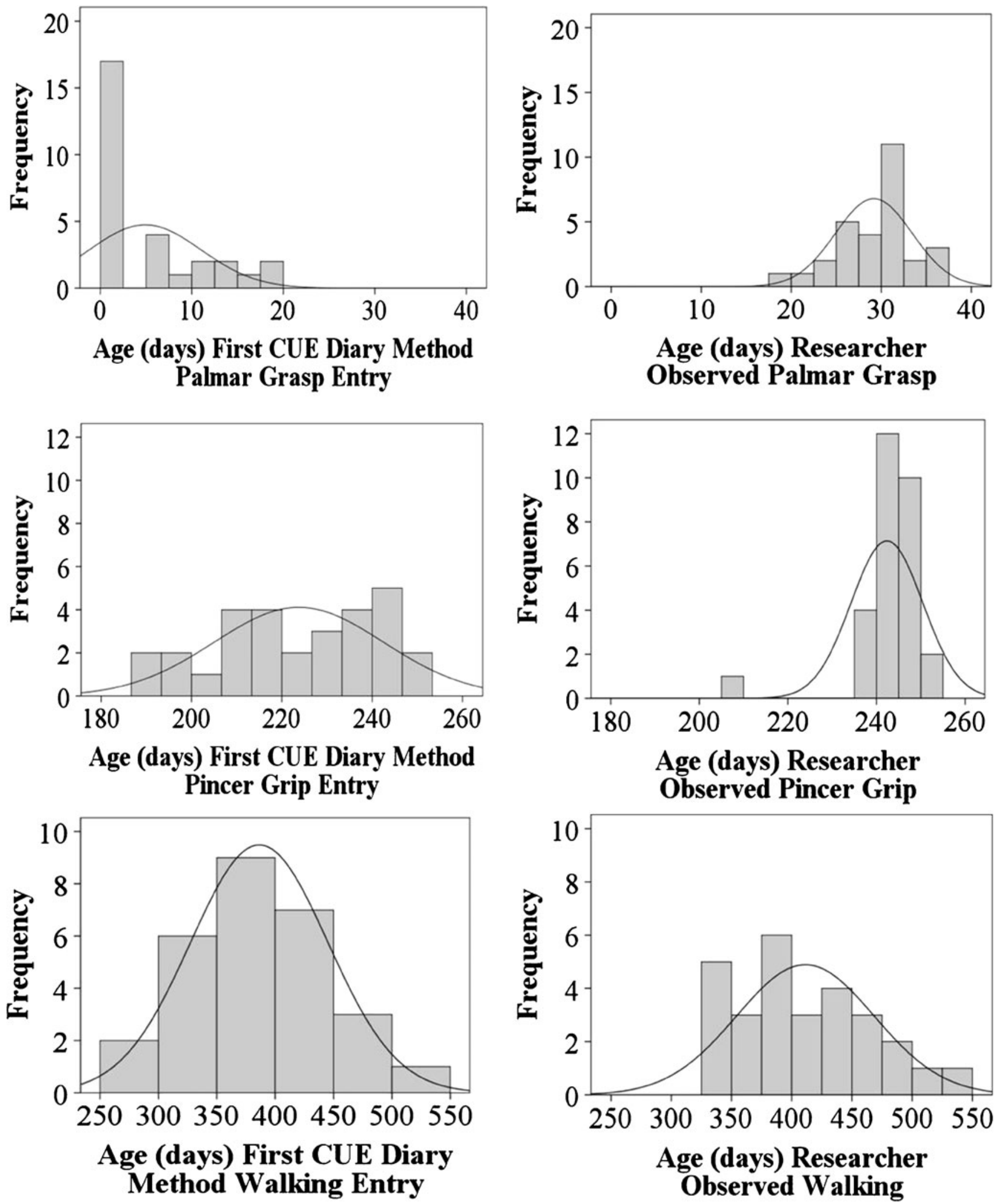

Fig. 3 Histograms demonstrating distortion in age of emergence from researcher assessments compared to continuous unified electronic (CUE) diary method data, with lines of best fit

child development. Diary data is valuable for the study of development because it draws on continuous observation and because it allows researchers to draw on rich contextual information. New parents have many demands on their time, and 
Table 3 Example entries for each of the target motor behaviors

\begin{tabular}{|c|c|c|c|}
\hline Question & Palmar Grasp & Pincer Grip & Walking \\
\hline Describe what happened & $\begin{array}{l}\text { Palmar grasped } \\
\text { mothers finger }\end{array}$ & $\begin{array}{l}\text { Baby is really dextrous now } \\
\text { and can pick up tiny objects } \\
\text { using a pincer grip }\end{array}$ & Baby took first steps unaided \\
\hline Describe where this happened. & At home during a feed & At home & Living room \\
\hline What type of behaviour was it? & Motoric & Motoric & Motoric \\
\hline What type of motoric behaviour was it? & Hand/Arm movement & Hand/Arm movement & Leg/Whole body movement \\
\hline Which motoric behaviour specifically? & Palmar grasp & Pincer grip & Took first steps \\
\hline Was this behaviour copied? & No & No & No \\
\hline $\begin{array}{l}\text { Was this behaviour spontaneous, } \\
\text { encouraged or instructed, or assisted? }\end{array}$ & Spontaneous & Spontaneous & Encouraged or instructed \\
\hline Describe how. & & & $\begin{array}{l}\text { I held baby upright and facing her } \\
\text { sister and said "you walk to your } \\
\text { sister now" }\end{array}$ \\
\hline $\begin{array}{l}\text { How many times has your baby } \\
\text { performed this behaviour? }\end{array}$ & This is the first time & This is the third time & This is the first time \\
\hline $\begin{array}{l}\text { If any objects were involved, } \\
\text { describe how. }\end{array}$ & & Little pieces of Lego bricks & $\begin{array}{l}\text { Baby carrying her sisters birthday } \\
\text { present }\end{array}$ \\
\hline
\end{tabular}

electronic diaries have previously been shown to offer greater ease of use than paper and pencil diaries (Lam et al., 2010). As a result, electronic diaries as a method encourage compliance and continued participation (Stone et al., 2002).

The CUE diary method allows researchers to gather contextual information using a combination of open-ended and closed-class questions. Understanding the role of context in shaping behavior is critical to understanding human development, and previous studies have shown that emergent behaviors are influenced by both physical and social environments (Collins, Maccoby, Steinberg, Hetherington, \& Bornstein, 2000). The CUE diary method can be used to identify age of emergence as well as the physical and social contexts in which behaviors emerge.

The CUE diary method is suitable for studying development across a range of domains. In the present study, we focused on motor behaviors because of their reliable elicitation once present in the infant's behavioral repertoire, and because we wanted to assess the reliability and value of the CUE diary method by comparing diary data with researcher assessments. Our complete CUE diary method included questions on cognitive and communicative development. The CUE diary method thus allows for the unified study of development across domains. Future research from our data set, for example, will examine the relations between motor and communicative development.

We used ESP software because it offered an easy-to-use platform with sufficient flexibility for our study, and installed the software on Palm Pilots. The CUE diary method is independent of the platform, however, and could easily be adapted for more recent versions of handheld computers, smartphones, or tablets.

\section{Training maternal reporters}

Previous electronic diary studies have, for the most part, involved self-report. Those studies that have involved parental reporting of infant behavior have focused on crying and sleeping, states that are both easily identified (Lam et al., 2010). To ensure that maternal reporters could remember and identify specific behaviors correctly, we developed a training and support system to accompany the electronic diaries. Future studies might implement these training components electronically, by using video training and other electronic resources.

Because the overall study involved comparisons across experimental and naturalistic data, the study design also included monthly visits to the university laboratory. Diary data was uploaded during these monthly meetings, and any immediate problems with the diaries were addressed. This regular contact may have had a positive effect on continued participation and compliance, but in future studies many of the conditions could be met by a combination of internetbased data collection and personal contact, which could include telephone conversations as well as electronic chats.

Feedback was also critical to the training and support system. Because the ESP software locks an entry as soon as it is completed, maternal reporters were not able to review previous questions or previous entries. To ensure that mothers knew which behaviors to watch for at any point in time, and to limit their dependence on memory for previous entries, entry feedback was given to mothers on a monthly basis. Following each upload of diary data, individual reports were created by researchers and sent to each maternal reporter. The reports summarized the past month's entries and gave a list of the yet-to-be-recorded target behaviors. Although other software could be configured to 
generate reports automatically, our researcher-led reports had the benefit of allowing researchers to screen for any incorrect entries and then reiterate behavioral definitions to reporters.

For future studies, the CUE Diary Method could be adapted for other designs with reduced training and support and larger sample sizes. Our training and support systems for maternal reporters required a substantial investment of researcher time. Further studies are needed to address whether similarly high levels of reliability could be achieved with reduced training and support.

\section{Assessing maternal reporter compliance}

Compliance evaluation focused on reporting of three motor behaviors: a reflex, a fine motor behavior, and a gross motor behavior. Our evaluation criteria concerned the timing of the entry, the quality of the entry, and the number of observations.

An important disadvantage of traditional paper-andpencil diaries is the impossibility of monitoring when entries are made, and in particular whether participants comply with time-related reporting instructions. We asked maternal reporters to create an entry for each target behavior as soon as possible after it had been observed. Because the CUE diary method included automatic time stamping of each entry, we were able to compare the reported date of an observed behavior with the actual entry date, and to disallow entries that were outside of an acceptable temporal threshold. Future studies could automate this evaluation procedure to reduce researcher time.

To further assess compliance, trained researchers reviewed each entry, checking whether the entry contained sufficient detail to identify the behavior, whether the entry met the definition provided for the behavior, and how many entries had been recorded for each behavior. The electronic format of the data facilitated this process by providing researchers with easy access to the entries in a spreadsheet format. We would not, however, recommend automating this checking procedure. The expert judgment of trained researchers was critical to this aspect of our evaluation, and provided the basis for feedback and further training. In future studies, this aspect of compliance evaluation could be handled through internetbased communications as well as telephone conversations.

\section{Assessing maternal reporter reliability}

Reliability was evaluated by comparing CUE diary data with researcher assessments for three target behaviors. Our evaluation criteria focused on whether maternal reporters identified behaviors at least as soon as a researcher identified them, and not more than two-months before that.

Our evaluation criteria were relatively strict. We required that each maternal reporter recorded each of our three motor milestones within this two-month window for any of her data to be included in future analyses. Our motivation for this stringent assessment method was that our overall data set also included other behaviors that we did not compare against researcher assessment. In order to be more confident about the reliability of those data, we applied a no-fails rule to the data where we did have researcher assessments. Over $80 \%$ of maternal reporters were deemed reliable, indicating that the CUE diary method is a valid and reliable method for collecting data on infant development.

Future studies using the CUE diary method could adopt one of two strategies for assessing reliability. A technology-based strategy would be to ask parents to provide documentary evidence of a select number of behaviors, in the form of photographs or videos. Researchers could then compare entries against documentary evidence to assess reliability. Because we used handheld devices that did not contain cameras, this option was not available to us, but because of recent increases in the availability, quality and affordability of smartphones with cameras, this should be a good option for the future.

A second strategy for assessing reliability in future studies would be to combine CUE diary data with researcher assessments, as we did, but to require fewer researcher assessments. In our present study, the majority of participants who failed reliability criteria for one target behavior also failed the reliability criteria for other target behaviors, suggesting that future studies adopting this strategy could use fewer researcher assessments to identify unreliable reporters. This strategy seems particularly useful for mixed-method studies in which the overall study design might require laboratory testing for other purposes.

\section{Advantages of electronic diaries when examining} developmental change

Our final aim was to compare CUE diary data with researcher assessments to establish the value of the CUE diary method. We were particularly interested in whether the CUE diary method produced a more accurate and detailed account of development. We found that the CUE diary method yielded greater detail of contextual information, earlier ages of emergence across motor behaviors, and a finer depiction of individual differences.

Studying the shape of developmental change requires an accurate tool for gathering information on the emergence of target behaviors. The CUE diary method combines the richness and flexibility of parent-report diary data with a diary method approximating the systematicity and rigor of experimental methods, and allows testing of causal hypotheses about human development. With appropriate piloting, the CUE diary method could also be adapted for the study of atypical development, including developmental delay. For example, the CUE diary method would be suitable for 
recording motor behaviors of infants whose motor development appears to be delayed, if training materials and support were designed for such samples. The contextual information contained in the CUE diary method, as well as the unified approach to studying development across domains, may be particularly useful for examining questions about developmental delay.

Limitations of electronic diaries for developmental studies

At present, electronic diary studies are expensive as compared with other forms of parent report, including checklists, questionnaires, and paper-and-pencil diaries. Because smartphones were not widely available when our study began, we provided every participant with a handheld computer, at a substantial cost per participant. In addition, our study procedures included extensive support for maternal reporters, and this support was time consuming, and therefore expensive. Importantly, however, the relative cost of electronic diary studies is likely to change quickly as technology changes. Future studies could adapt the CUE diary method for smartphones and might even rely on participant-owned devices. Future studies might also use electronic resources for training and feedback to reduce costs. For example, video training and electronic messaging could be used for some of the procedures that involved one-on-one staff time in our study.

In our present study, some motor behaviors appeared easier for maternal reporters to accurately identify and record than others. For example, comparison of the earliest entries for walking and climbing stairs suggests that some maternal reporters may have been recording climbing stairs inaccurately, for instance, recording a scaffolded version of climbing stairs, rather than independent, upright climbing. In comparison, CUE data for all six of the remaining motor behaviors showed emergence within the range expected, and in the order of emergence expected. Similarly, the three behaviors of palmar grasp, pincer grip, and walking, all showed high levels of reliability, as predicted. Future studies would need to include piloting to ensure clear definitions which support accurate reporting of complex behaviors.

\section{Conclusions}

The CUE diary method is the first adaptation of event-based electronic diary methods for the study of infant and child development. Like historical parent-report diaries, the CUE diary method is based on continuous observation and provides data about infants in their natural environment. As a result, the CUE diary method allows researchers to collect data on age of emergence and important contextual variables such as precursor variables, eliciting factors, and rewards.
Unlike traditional paper-and-pencil diaries, the CUE diary method allows for larger samples and reduced attrition because it is easier and quicker for participants to use due to menus, data uploading capabilities, and other time-saving features. In addition, it facilitates assessment of compliance and reliability. Through its adaptable design, the CUE diary method may be applied to other hand-held devices such as smartphones in the future.

Kuhn (1995) argued that developmental psychology aims to see the process of change, rather than simply its products. The CUE diary method is an excellent methodological tool for investigating the mechanisms of change in human development and yields event-driven, context-rich, longitudinal data across development. The CUE diary method opens the door to examining the dynamic nature of human development.

Author note Thanks to Malinda Carpenter and Ulrike Hahn for their valuable discussions during this project. Elena Sakkalou is now working at the Institute of Child Health, University College London. Elma Hilbrink is now working at the Max Planck Institute for Psycholinguistics, Nijmegen. The present research was supported by the Leverhulme Trust and the Economic and Social Sciences Research Council. Address correspondence to M. Gattis, School of Psychology, Cardiff University, Tower Building, Park Place, Cardiff. CF24 3AT (e-mail: gattism@cardiff.ac.uk).

\section{Appendix A}

First steps handbook

\section{Introduction}

By now we will have met with you and will have given you your own Palm. We will have tried our best to explain how to use it, and what we expect from you and your baby during this First Steps study. Since our meeting, your bundle of joy will have arrived and everything will have suddenly become very hectic and disorganized! With all this commotion, it would be highly unusual if you remembered everything that we have told you. This guide is meant to refresh your memory and provide some useful hints and tips.

\section{What is expected of me?}

We would like you to complete the brief set of questions on your Palm as soon as your infant performs any of the new behaviours that we are interested in. Please note that we would like you to record only the first three times that your infant performs each behaviour. Examples of the behaviours that we are interested in are: when your infant copies your actions, sounds, facial expressions, gestures, uses objects, and when your infant reaches specific motor milestones. 
The sooner the questions are completed after your infant has performed a behaviour the better. This helps to ensure that you are able to provide the richest description of what has happened. The information that you provide us with, by answering these questions, will form the basis of a "memory book" which we will give you as a gift at the end of the study. This "memory book" will contain details about your baby's firsts... the first time your baby walked, talked, and copied you. Therefore, the more details that you can remember, the better equipped we will be to try and preserve the memories of your baby's firsts in the "memory book". It will also help ensure that the data that we gather is as accurate as possible.

We would like to meet with you once a month for brunch, on a Friday at Café Junior. Other mothers and their babies who are taking part in the First Steps study will also be there. This will give you the chance to socialize with other mums who have babies of a similar age and enjoy a change in scenery. During this meeting we will have a chat with you and check that you have not encountered any problems. We will download the information that you have been recording for us from your Palm onto our computer. During this session we will also want to see how your infant responds to certain things, for example, their reactions to faces, sounds, toys, and actions such as grasping, sitting up, and walking. These meetings will last between one to two hours and they are critical to the study, so please try your very best to make these meetings. At every third meeting, we will give you a "what to expect sheet" detailing which specific behaviours we want you to look out for over the following months. We will also give you a toy or book for your child, and a $£ 25$ gift voucher to thank you for helping us.

We will phone you once a week to have a chat and see if everything is going okay. During our call we will discuss how you are finding things and whether you have encountered any problems. We will also remind you when your next meeting will take place at Café Junior. However, if you encounter any problems before this weekly phone call, please contact us. To maximize consistency for you, we are going to pair you with a key researcher. Therefore, the person who initially starts corresponding with you will be the person who continues to keep in touch with you over the course of the study. This will be the person you should contact if you encounter any problems or have any questions.

\section{How do I use the Palm pilot?}

Before your baby arrives it is a good idea to practise using your Palm. It would be useful to practise writing using the touch screen. The fastest and easiest way to write on the Palm is to use the touchscreen keyboard. To access the keyboard, tap on the $\mathrm{ABC}$ icon on the bottom left of the screen. This will allow you to complete text response questions using a keyboard that will appear on the touch screen. Simply tap a letter to insert it in your entry.

Alternately you can write using strokes on the graffiti pad at the bottom of the screen. Each letter of the alphabet is assigned a specific stroke. Most of these strokes are the same as you would use when writing normally but there are a few tricky ones. There is a very useful sticker on the back of your Palm illustrating the different strokes required to write each letter of the alphabet, punctuation, and numbers. This guide (see page 7) states whether you should perform the strokes on the left or the right graffiti pad. Please note that letters and the most common types of punctuation need to be written on the left graffiti pad (eg., ? “"). Numbers and the more complex forms of punctuation (eg $/ \backslash())$ need to be written on the right graffiti pad. This guide also illustrates which strokes will create a space between words, and which will delete a single letter. A space can be created between words by making a forward horizontal stroke on the left graffiti pad, whereas, you can delete a single letter by making a backward stroke on the left graffiti pad.

It would also be a good idea to practise recording behaviours on your Palm. For instance, if you have another child you could complete the brief series of questions based on behaviours that he or she exhibits, or alternatively, note behaviour that your partner performs. Practice recording behaviours on the Palm will give you an impression of what details to look out for when your baby performs a behaviour. It will also allow you to identify any problems that you may encounter, so that they can be rectified before your baby arrives. If you practise completing these questions before your baby arrives, please note down on the final question ("If you feel you have made any mistakes in completing this questionnaire, or you would like to change anything, please note it here.") that it is a practice.

To record what behaviour your infant has performed, please turn the palm on, and tap the "esp" icon on the main menu. This will start a brief series of questions that will help you to recall the specific details of your infant's behaviour. There are three different types of questions that you will be asked; button response questions, pop-up response questions, and text response questions. With button response questions, you just need to tap the button on the touch screen that seems most 
relevant. With pop-up response questions, you need to tap on "click to choose a response" on the touch screen. This will display a pop-up menu where you can tap on the response that is most relevant. Once you have done this, you will need to tap on the "done" button in the bottom left corner of the touch screen. With text response questions, you may need to refer to the stroke guide on the back of your Palm. Use the left graffiti pad, or keyboard, to write your brief text response. Text response questions are limited to just 120 characters. When you have finished writing, tap the "done" button in the bottom left corner of the touch screen.

It is important that you complete all questions until the screen reads "Questionnaire completed. Thank you". Unless you answer all the questions the Palm will not save what you have recorded. Once the questionnaire is complete you can exit by tapping on the clock icon in the bottom left graffiti pad. This will bring up a screen which will have the option "go to clock". Tap on "go to clock" and once you are in the clock, tap on the home icon on the left graffiti pad. This will bring you back to the main menu. Once on the main menu, you can safely turn the Palm off.

\section{Looking after my Palm}

Please charge your Palm every evening. This will ensure that the battery will not run out at a crucial time when your infant is performing a behaviour, or achieving a milestone for the first time.

It is very important that you do not use the memo function on the Palm as it will interfere with the questions that are programmed into the Palm, and these questions are vital to the experiment.

You are free to use the diary and contacts applications, but we ask that you not put photographs on the Palm. Storing photographs puts a high demand on the Palm's limited memory and could mean that there is no memory left for you to record the behaviours your infant performs we would miss out on information about some of the crucial steps in your child's development.

Please do not connect your Palm to a computer. Your Palm is set to be synchronised to one of our computers. If you connect your Palm to another computer during the course of the study it may confuse the Palm. This could result in the information that you have recorded being erased, and the questionnaire that is programmed into the Palm being altered.

Try your best to keep your Palm away from water and out of direct sunlight. However, accidents do happen, especially when your infant becomes mobile and likes to hide or drop things. Please let us know as soon as possible if anything happens to your Palm.

\section{Troubleshooting with the Palm Pilot}

Once you have started filling in the series of questions, please try your best to complete them. There is purposely no direct exit button as we want to encourage people to complete all the questions. However, if you have made a mistake, you can exit the questionnaire relatively quickly, without affecting the information that you have already recorded. Simply selecting "done" for responses to the text entry questions without writing an answer, and by selecting any of the button or pop-up answers. If you use this method it is vital that you complete the final question. This final question asks you whether you feel you have made any mistakes in completing the questionnaire. Please note down what mistake you have made. This final question will allow us to identify that the information you have entered is incorrect and that we should disregard it.

If you encounter any problems that prevent you from recording your infant's behaviour on your Palm, please contact us as soon as possible and do not wait for our next meeting. We will try our best to sort problems out as quickly as possible.

Our goals can be summarised in two steps.

1) Observing your baby. You can help us by observing your baby's behaviour closely. Most parents find this comes quite naturally!

2) Telling us about your baby. Please record each observation of your baby's behaviour in your Palm diary as soon as possible after you observe it. Please provide us with as much detail as you can. Doing so will help us understand the details of what your baby did, where it happened, and how it happened. It will also help to create a more rewarding "memory book" for you at the end of our study!

The first two questions ask you to provide a general description of what happened, and where it happened. Your answers don't need to be long, but they should provide a recognisable summary of the behaviour. You might want to think of the first two questions as a snapshot memory for your memory book.

Diary tip Each text entry has a limit of 120 characters. This is about one long sentence or two short sentences.

After telling us about what happened and the context where it happened, you will be asked whether the behaviour you are recording was communicative, imitative, or motoric. On the following pages we provide information about questions within each of those categories. 
Diary tip You may find it easier and faster to use the touchscreen keyboard for text entry. Just tap on the ABC icon to access the keyboard.

\section{Appendix B}

Motoric behaviours

\section{Which behaviour?}

To make diary records easier and faster for you, we have divided the motoric behaviours we would like you to record into two questions. First you need to choose whether the movement involved the hands and arms or the legs or whole body. Then you need to choose the specific behaviour from a list.

Hand/arm movement

- palmar grasp

- picked up small objects

- reached for object when lying on front

- reached for object when sitting

- turned multiple pages of a book

- pincer grip

- scribbled spontaneously

- put object in container spontaneously

Leg/whole body movement

- started crawling

- turned from lying on side to lying on back

- stands unsupported

- took first steps

- climbed stairs

Diary tip We will provide you with more detailed information about key motoric behaviours in the "What to Expect" sheets. You only need to watch for a particular motoric behaviour once it has been presented in one of your "What to Expect" sheets.

\section{Was the behaviour copied?}

If you or someone else modelled the behaviour for your baby, and your baby's behaviour followed closely after, your baby may have copied the behaviour. This is going to make more sense with some behaviours than others. For instance, even though your baby may have seen you walking just before taking his or her first steps, you would not consider those first steps to be copied.

\section{What else was involved in the behaviour?}

In the following questions we will ask you to provide details about whether you assisted or encouraged your baby, how many times your baby has performed this behaviour, and whether any objects were involved. We are only asking you to record a behaviour the first three times your infant does it. If you cannot remember how many times your baby did the recorded behaviour, make your best guess. We can check this against the previous diary entries.

\section{Appendix C}

What to expect from your baby girl from birth to 3 months

\section{Motoric development}

From birth your daughter will most likely be able to grasp a finger (surprisingly strongly!) when her palm is touched. She will probably show signs of rooting and sucking. If her cheek is touched at the corner of her mouth, she may turn to that side and try to suck the finger.

By the first month, although your daughter's eyesight is still in the initial stages of development, for a short time she will probably be able to follow with her eyes a large object that is moving on a slow and predictable path.

Your daughter may start to show an interest in her hands. She may start to inspect her hands, bringing them in front of her face, and later play with them (flexing and unflexing her fingers). This is often referred to as "Finger Play". At around 3 months, she may even start trying to pick up small objects, such as a cube.

When lying on her front, or back, her position may gradually loosen, changing from being in a tightly curled up foetal position, with knees and arms tucked in towards her body, to stretching out with legs and arms extended, or with arms gently relaxed by the side of her body.

Over the course of the first 3 months, the muscles in your daughter's neck and back will likely strengthen. She may become able to roll from lying on the side to lying on the back. She may be able to sit up whilst being held, and gradually be able to hold the head and chin steady, unsupported, for several seconds.

Developmental milestones

Palmar grasp - object or finger is held in palm with a strong grasp. 


\section{References}

Adolph, K. E., \& Robinson, S. R. (2008). In defense of change processes. Child Development, 79, 1648-1653. doi:10.1111/j.14678624.2008.01215.x

Adolph, K.E., Robinson, S.R., Young, J.W., \& Gill-Alvarez, F. (2008). What is the shape of developmental change? Psychological Review, 115 .

Alpers, G. W. (2009). Ambulatory assessment in panic disorder and specific phobia. Psychological Assessment, 21, 476-485. doi:10.1037/ A0017489

Bakeman, R. (1983). Theory and practice of observing behavior Fassnacht, G. Contemporary Psychology, 28, 589-590.

Bodnarchuk, J. L., \& Boudreau, J. P. (2004). Can parents be trusted? Validity of daily checklists of gross motor milestone attainment. Applied Developmental Psychology, 25, 481-490.

Bolger, N., Davis, A., \& Rafaeli, E. (2003). Diary methods: Capturing life as it is lived. Annual Review of Psychology, 54, 579-616. doi:10.1146/annurev.psych.54.101601.145030

Bornstein, M. H. (1985). How infant and mother jointly contribute to developing cognitive competence in the child. Proceedings of the National Academy of Sciences, 82, 7470-7473.

Callanan, M. A., \& Oakes, L. M. (1992). Preschoolers' questions and parents' explanations: Causal thinking in everyday activity. Cognitive Development and Psychopathology, 7, 213-233.

Collins, W. A., Maccoby, E. E., Steinberg, L., Hetherington, E. M., \& Bornstein, M. H. (2000). Contemporary research on parenting. The case for nature and nurture. American Psychologist, 55, 218-232.

Cronbach, L. J. (1957). The two disciplines of scientific psychology. American Psychologist, 12, 671-684.

Danis, A., Bourdais, C., \& Ruel, J. (2000). The co-construction of joint action between mothers and 2-4-month-old infants: The mother's role. Infant and Child Development, 9, 181-198.

Danziger, K. (2000). Making social psychology experimental: A conceptual history, 1920-1970. Journal of the History of the Behavioral Sciences, 36, 329-347.

Dekker, M. C., Nunn, R. J., Einfeld, S. E., Tonge, B. J., \& Koot, H. M. (2002). Assessing emotional and behavioral problems in children with intellectual disability: Revisiting the factor structure of the developmental behavior checklist. Journal of Autism and Developmental Disorders, 32, 601-610. doi:10.1023/a:1021263216093

Dunn, W. (1990). Establishing inter-rater reliability on a criterionreferenced developmental checklist. Occupational Therapy Journal of Research, 10, 377-380.

Ebner-Priemer, U.W., Kuo, J., Kleindienst, N., Welch, S.S., Reisch, T., Reinhard, I., . . . Bohus, M. (2007). State affective instability in borderline personality disorder assessed by ambulatory monitoring. Psychological Medicine, 37, 961-970. doi:10.1017/S0033291 706009706

Ebner-Priemer, U. W., \& Trull, T. J. (2009). Ambulatory assessment: An innovative and promising approach for clinical psychology. European Psychologist, 14, 109-119. doi:10.1027/1016-9040.14.2.109

Ebner-Priemer, U. W., \& Sawitzki, G. (2007). Ambulatory assessment of affective instability in borderline personality disorder - The effect of the sampling frequency. European Journal of Psychological Assessment, 23(4), 238-247. doi:10.1027/1015-5759. 23.4.238

Fahrenberg, J., Myrtek, M., Pawlik, K., \& Perrez, M. (2007). Ambulatory assessment-monitoring behavior in daily life settings-A behavioral-scientific challenge for psychology. European Journal of Psychological Assessment, 23, 206-213. doi:10.1027/10155759.23.4.206

Feldman Barrett, L., \& Barrett, D. J. (2001). An introduction to computerized experience sampling in psychology. Social Science Computer Review, 19, 175-185.
Fenson, L., Pethick, S., Renda, C., Cox, J. L., Dale, P. S., \& Reznick, J. S. (2000). Short-form versions of the MacArthur Communicative Development Inventories. Applied PsychoLinguistics, 21, 95-115.

Gartstein, M. A., \& Rothbart, M. K. (2003). Studying infant temperament via the Revised Infant Behavior Questionnaire. Infant Behavior \& Development, 26, 64-86.

Green, A. S., Rafaeli, E., Bolger, N., Shrout, P. E., \& Reis, H. T. (2006). Paper or plastic? Data equivalence in paper and electronic diaries. Psychological Methods, 11, 87-105. doi:10.1037/1082989X.11.1.87

Gunthert, K. C., Conner, T. S., Armeli, S., Tennen, H., Covault, J., \& Kranzler, H. R. (2007). Serotonin transporter gene polymorphism (5-HTTLPR) and anxiety reactivity in daily life: A daily process approach to gene-environment interaction. Psychosomatic Medicine, 69(8), 762-768. doi:10.1097/Psy.0b013e318157ad42

Holmes, A., \& Teti, D. M. (2008). Developmental science and the experimental method handbook of research methods in developmental science (pp. 15-80): Blackwell Publishing Ltd.

Hoppmann, C. A., \& Riediger, M. (2009). Ambulatory assessment in lifespan psychology: An overview of current status and new trends. European Psychologist, 14, 98-108. doi:10.1027/10169040.14.2.98

Hufford, M. R., \& Shields, A. L. (2002). Electronic diaries. Applied Clinical Trials, 11, 46-52.

Jamison, R. N., Raymond, S. A., Levine, J. G., Slawsby, E. A., Nedeljkovic, S. S., \& Katz, N. P. (2001). Electronic diaries for monitoring chronic pain: 1-year validation study. Pain, 91, 277-285.

Johnson, K. E., \& Mervis, C. B. (1997). First steps in the emergence of verbal humor: A case study. Infant Behavior \& Development, 20 , $187-196$.

Kagan, J. (2008). In defense of qualitative changes in development. Child Development, 79, 1606-1624.

King, N. A., Lluch, A., Stubbs, R. J., \& Blundell, J. E. (1997). High dose exercise does not increase hunger or energy intake in free living males. European Journal of Clinical Nutrition, 51, 478483.

Klumb, P., Elfering, A., \& Herre, C. (2009). Ambulatory assessment in industrial/organizational psychology: Fruitful examples and methodological issues. European Psychologist, 14, 120-131. doi:10.1027/1016-9040.14.2.120

Kuhn, D. (1995). Microgenetic study of change: What has it told us. Psychological Science, 6, 133-139.

Lam, J., Barr, R. G., Catherine, N., Tsui, H., Hahnhaussen, C. L., Pauwels, J., \& Brant, R. (2010). Electronic and paper diary recording of infant and caregiver behaviors. Journal of Developmental and Behavioral Pediatrics, 31, 685-693.

Lewis, M. D. (2000). The promise of dynamic systems approaches for an integrated account of human development. Child Development, $71,36-43$

McCall, R. B. (1981). Nature-nurture and the two realms of development- A proposed integration with respect to mentaldevelopment. Child Development, 52, 1-12.

Miller, S. (1981). Assessing language production in children. Baltimore, MD: University Park Press.

Mitchell, S. K. (1979). Interobserver agreement, reliability, and generalizability of data collected in observational studies. Psychological Bulletin, 86, 376-390.

Muller, U., \& Giesbrecht, G. (2008). Methodological and epistemological issues in the interpretation of infant cognitive development. Child Development, 79, 1654-1658. doi:10.1111/j.14678624.2008.01216.x

Myrtek, M., Aschenbrenner, E., \& Brugner, G. (2005). Emotions in everyday life: An ambulatory monitoring study with female students. Biological Psychology, 68(3), 237-255. doi:S0301051 104001036 [pii]10.1016/j.biopsycho.2004.06.001 
Naigles, L. R., Hoff, E., \& Vear, D. (2009). Flexibility in early verb use: Evidence from a multiple-N diary study. Monographs of the Society for Research in Child Development, 74, 1-143.

Nelson, K. (Ed.). (1989). Narratives from the crib. Cambridge, MA: Harvard University Press.

Pine, J. M. (1995). Variation in vocabulary development as a function of birth order. Child Development Perspectives, 66, 272-281.

Reznick, J. S., \& Goldfield, B. A. (1992). Rapid change in lexical development in comprehension and production. Developmental Psychology, 28, 406-413. doi:10.1037/00121649.28.3.406

Reznick, J. S., \& Goldfield, B. A. (1994). Diary vs. representative checklist assessment of productive vocabulary. Journal of Child Language, 21, 465-472.

Robinson, B. F., \& Mervis, C. B. (1999). Comparing productive vocabulary measures from the CDI and a systematic diary study. Journal of Child Language, 26, 177-185.

Seifer, R. (2005). Who should collect our data: Parents or trained observers? In D. M. Teti (Ed.), Handbook of research methods in developmental science (pp. 123-137). Malden, MA: Balckwell.

Stone, A. A., Broderick, J. E., Kaell, A. T., DelesPaul, P. A. E. G., \& Porter, L. E. (2000). Does the peak-end phenomenon observed in laboratory pain studies apply to real-world pain in rheumatoid arthritics? The Journal of Pain, 1, 212-217.

Stone, A. A., Shiffman, S., Schwartz, J. E., Broderick, J. E., \& Hufford, M. R. (2002). Patient non-compliance with paper diaries. British Medical Journal, 324(7347), 1193-1194.

Tomasello, M. (1992). First verbs: A case study of early grammatical development. Cambridge, England: Cambridge University Press.

Wijnhoven, T. M. A., de Onis, M., Onyango, A., Wang, T., Bjoerneboe, G. E. A., Bhandari, N., \& Al Rashidi, B. (2004). Assessment of gross motor develoment in the WHO Multicentre Growth Reference Study. Food and Nutrition Bulletin, 25, 537-545.

Wolfson, A., Lacks, P., \& Futterman, A. (1992). Effects of parent training on infant sleeping patterns, parents' stress, and perceived parental competence. Journal of Consulting and Clinical Psychology, 60, 41-48. doi:10.1037/0022-006x.60.1.41 